\title{
Polyesters by a Radical Pathway: Rationalization of the Cyclic
} Ketene Acetal Efficiency

\author{
Antoine Tardy, Noémie Gil, Christopher M. Plummer, Didier Siri, Didier Gigmes, Catherine Lefay, ${ }^{*}$ \\ Yohann Guillaneuf*
}

[a] Dr. A. Tardy, N. Gil, Dr. C. M. Plummer, Pr. D. Siri, Dr. D. Gigmes, Dr. C. Lefay, Dr. Y. Guillaneu

Aix-Marseille Université, CNRS

Institut de Chimie Radicalaire, UMR 7273

Marseille, France

E-mail: yohann.guillaneuf@univ-amu.fr

Supporting information for this article is given via a link at the end of the document

\begin{abstract}
Radical Ring-Opening polymerization (rROP) of Cyclic Ketene Acetals (CKAs) combines the advantages of both ringopening polymerization and radical polymerization thereby allowing the robust production of polyesters coupled with the mild polymerization conditions of a radical process. rROP was recently rejuvenated by the possibility to copolymerize CKAs with classic vinyl monomers leading to the insertion of cleavable functionality into a vinyl-based copolymer backbone and thus imparting (bio)degradability. Such materials are suitable for a large scope of applications, particularly within the biomedical field. The competition between the ring-opening and ring-retaining propagation routes is a major complication in the development of efficient CKA monomers, ultimately leading to the use of only four monomers that are known to completely ring-open under all experimental conditions. In this article we investigate in detail the radical ring-opening polymerization of model CKA monomers and demonstrate by the combination of DFT calculations and kinetic modeling using PREDICI software that we are now able to predict in silico the ring-opening ability of CKA monomers.
\end{abstract}

\section{Introduction}

Recently, the final outcome of synthetic polymers has become a major issue due to the accumulation of wastes that are damaging the environment. ${ }^{[1-3]}$ To overcome this problem the use of biodegradable polymers such as polyesters, in particular polylactides, has been widely investigated. ${ }^{[4-6]}$ These polymers are commonly prepared by polycondensation or Ring-Opening Polymerization (ROP). ${ }^{[7]}$ Nevertheless, a large part of commodity and specialty polymers are produced by radical polymerization techniques that impede the introduction of ester linkages into the backbone of the polymer chains. ${ }^{[8]}$ Relatively few studies, ${ }^{[9-10]}$ initiated by the pioneering work of Bailey et al. ${ }^{[11]}$, have demonstrated that various cyclic monomers bearing vinyl or exomethylene groups could be polymerized radically through a ring-opening process (Scheme 1a).

Radical Ring-Opening Polymerization (rROP) presents the advantages of both ring-opening polymerization and radical polymerization, specifically the production of (co)polymers with the possibility to insert heteroatoms and/or functional groups into the main chain by facile polymerization procedures (Scheme 1a). ${ }^{[10]}$ The ring-opening polymerization of Cyclic Ketene Acetals (CKAs) by free radical or controlled radical mechanisms has attracted considerable interest since it presents an alternative route for the synthesis of functionalized aliphatic polyesters ${ }^{[12-15]}$ and is a powerful tool for imparting degradability to vinyl polymers ${ }^{[16-20]}$ by introducing ester linkages into their backbone (Scheme 1b). ${ }^{\left[{ }^{[0-10]}\right.}$

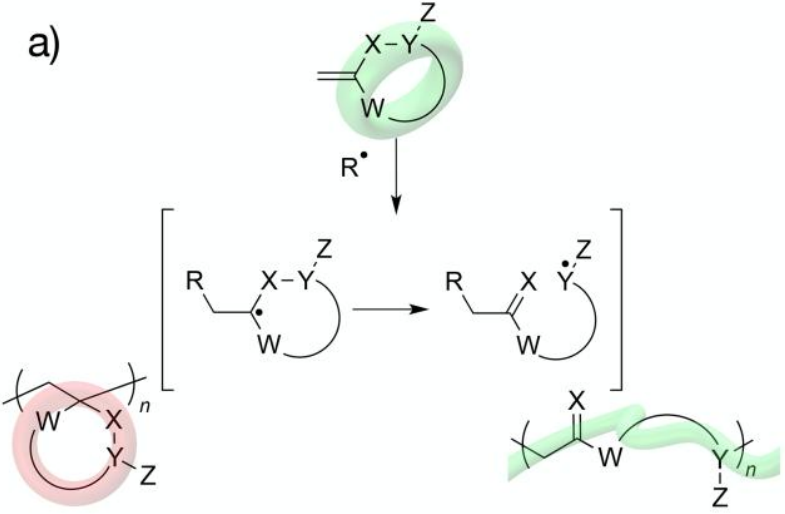

b)
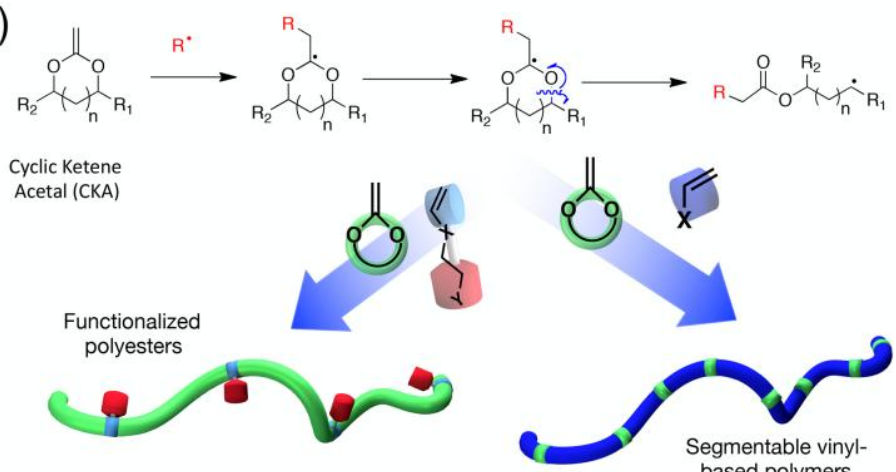

Scheme 1. a) Mechanism of Radical Ring-Opening Polymerization (rROP). b) rROP of Cyclic Ketene Acetals (CKAs) and their use in the preparation of functionalized polyester or segmentable vinyl-based polymers. 
Indeed, the copolymerization of vinyl-based monomers and CKAs has been used to prepare (bio)degradable polyethylene, ${ }^{[21]}$ fluoropolymers, ${ }^{[2]}$ polymethyl methacrylate materials ${ }^{[18]}$ marine anti-biofouling coatings, ${ }^{[23-24]}$ etc., offering a promising route to reduce plastic pollution. This approach has also been used to confer (bio)degradability to many vinyl-based biomaterials such as polymer prodrugs, ${ }^{[25]}$ nanoparticles, ${ }^{[26-27]}$ thermo-responsive materials, ${ }^{[20,}{ }^{28-29]}$ ice-recrystallization inhibitors, ${ }^{[30]}$ etc. To confer efficient (bio)degradability, the incorporation of ester bonds into the polymer backbone has to be performed randomly, as demonstrated theoretically by Lefay et al. ${ }^{[31]}$ using kinetic modelling. Even though the rROP has been known since the eighties, very few mechanistic studies have been performed and no clear structure-reactivity relationships have been established for those monomers. ${ }^{[10,32-33]}$ Indeed, the major drawback of this family of monomer is related to the competition between the ring-opening process and the undesired direct vinyl propagation that leads to polyacetal (Scheme 2). Thus, for the majority of CKA monomers, the resultant polymer is a random copolymer of ester and acetal units.
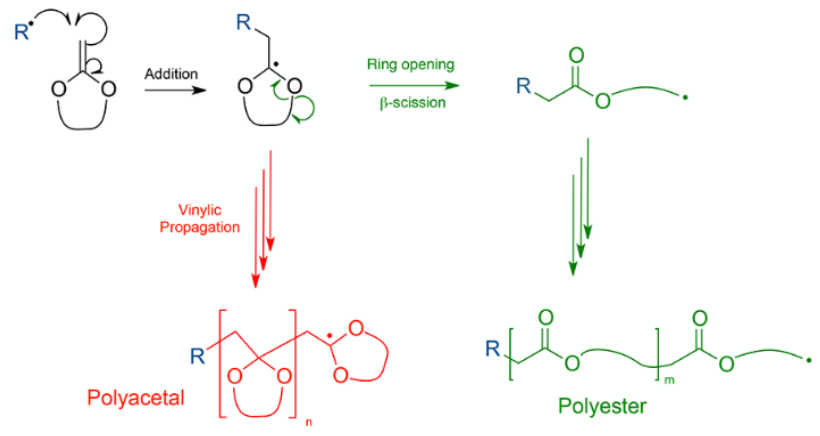

Scheme 2. Competition between ring-opening and direct vinylic propagation leading to either ester units or acetal units.

This situation leads to the predominant use of two cyclic monomers in all of the literature; 2-methylene-1,3-dioxepane (MDO, C7, Scheme 3) and the 5,6-benzo-2-methylene-1,3dioxepane (BMDO, C7B, Scheme 3), both known to undergo complete ring-opening polymerization independent of the conditions. ${ }^{[10]}$ Promising results have been obtained for biomedical applications with these two monomers (gene transfection, etc.). ${ }^{[10]}$ The development of new CKA monomers that can extend the properties of the corresponding polyester by tuning the mechanical properties, degradation rate, etc. is paramount. To design a monomer for radical ring-opening polymerization, originally two factors were taken into account in order to improve the selectivity of the ring-opening process: (i) the ring strain and (ii) the stabilization of the ring-opened radical. For example, 6-membered ring monomers are recognized to be difficult to polymerize via a ring-opening process. This is usually explained by the low ring-strain of monomers analogous to cyclohexane (whose ring-strain is set to zero). The addition of an aromatic ring close to one oxygen (2 methylene-4-phenyl-1,3dioxane, C6P, Scheme 3) was also expected to increase the ring-opening ability via the stabilization of the released alkyl radical, but the radical polymerization of such monomers, under both UV at $25-50{ }^{\circ} \mathrm{C}$ and thermal initiation at 80 and $120{ }^{\circ} \mathrm{C}$, gave polyacetals of high molar mass $\left(>30,000 \mathrm{~g} \cdot \mathrm{mol}^{-1}\right){ }^{[34]}$ On the contrary, an analogous chemical modification of the 5membered monomer (2-methylene-4-phenyl-1,3-dioxolane or MPDL, C5P, Scheme 3) increases the ring-opening ability. ${ }^{[35-36]}$ There are many similar examples showing that the aforementioned factors were not key parameters for rationalizing the reactivity of such monomers. ${ }^{[10]}$

These results demonstrate why only a few known monomers are used within the literature and why the versatility of the rROP technique to prepare a wide range of functionalized polyesters has not been extensively investigated. In order to efficiently design new monomers, it would be useful to be able to predict the efficiency of a given monomer in rROP before its synthesis and polymerization. Herein, we propose a theoretical approach that combines quantum calculations and kinetic modeling based on the competitive process described above (Scheme 2) to achieve this goal.

More precisely, our strategy is to use Density Functional Theory (DFT) to calculate the various rate coefficients that will be later implemented into a kinetic model to obtain the theoretical composition of ester and acetal units in a polymer backbone. These theoretical results were then experimentally confirmed by studying the radical polymerization of $\mathbf{C 5}, \mathbf{C 5 H}, \mathbf{C 6}$ and $\mathbf{C 7}$ CKA monomers (Scheme 3). Such an approach allows the in silico prediction of the behavior of new CKA monomers.
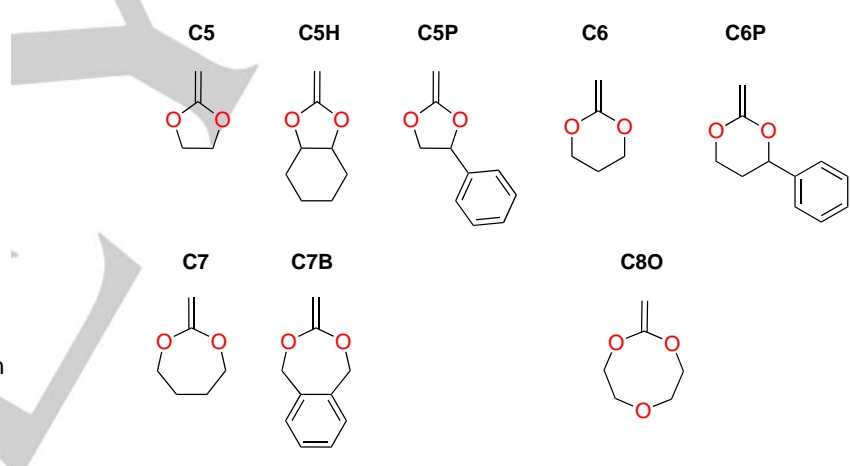

C80
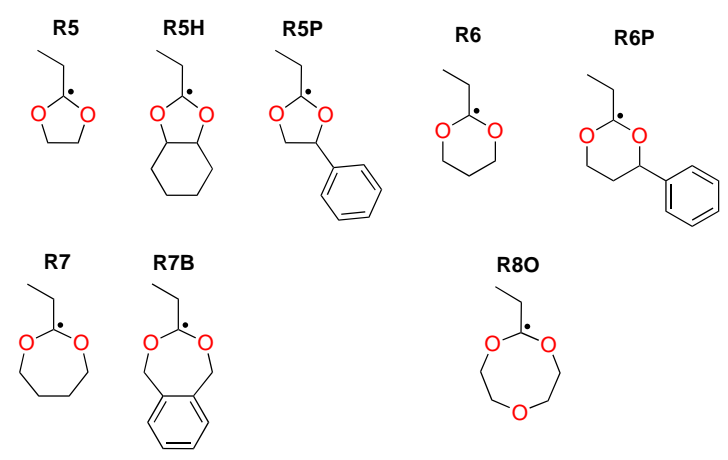

Scheme 3. Structures of CKA monomers and their corresponding intermediate acetalyl radicals.

\section{Results and Discussion}

\section{Experimental Study}


A significant problem when rationalizing the reactivity of CKA monomers arises from the many experimental conditions that have been reported within the literature (initiator nature and concentration, temperature, etc.). Additionally, since many of the published studies were performed in the $80-90$ s, some nonreliable characterization methods have been used to assess the ratio between acetal and ester repeating units. To ensure the validity of the experimental data before implementing the modeling, we performed the polymerization of monomers C5, C5H, $\mathbf{C 6}$ and $\mathbf{C 7}$ coupled with careful microstructure analysis to obtain an accurate ratio between the acetal and ester units for these model CKA polymers.

As was previously stated by Bailey et al ${ }^{[11]}$ in their pioneering studies, CKA monomers with 5- and 6-membered rings are prone to cationic polymerization initiated by contact with glass making purification steps particularly problematic. This is a crucial issue when attempting to quantify the ester percentage obtained by radical polymerization as cationic polymerization typically results in a high quantity of acetal units, or even pure polyacetal (see ESI Fig. S1). Thus, spontaneous cationic polymerization contamination during monomer purification, storage, radical polymerization and subsequent analysis was avoided through strict experimental conditions. The bulk polymerizations were performed using $3 \mathrm{~mol} \%$ of the various radical initiators depending on the polymerization temperature (see ESI for details). After the polymerization of monomers C5, $\mathrm{C} 5 \mathrm{H}, \mathrm{C6}$ and $\mathbf{C 7}$ and purification by precipitation, the four polymers were analyzed by ${ }^{1} \mathrm{H}$ and ${ }^{13} \mathrm{C}$ NMR. All ${ }^{1} \mathrm{H}$ and ${ }^{13} \mathrm{C}$ NMR signals can be assigned and are consistent with the data reported within the literature. 2D NMR techniques also confirmed these assignments (see ESI for details, Figure S2 and S3 for PC5 for example). The analysis of the ${ }^{13} \mathrm{C}$ NMR spectra (Fig. 1 for the polymerization of monomer C5, other NMR spectra are provided in ESI) confirmed the presence of two repeating units within the copolymers. These spectra display peaks in the characteristic zones of both acetal and ester units, at 100 and $170 \mathrm{ppm}$, respectively. We also noted a change in the number (i.e. the presence of diads, see Figure 1) and intensity of these peaks corresponding with the temperature of polymerization. The acetal peaks were more intense at low temperatures, while there was an inversion in the intensity of the two main ester peaks with temperature. It is clear that an increase in temperature promotes the presence of ester units, which are highly prevalent at $150^{\circ} \mathrm{C}$. After full assignment of the ${ }^{1} \mathrm{H}$ NMR spectrum, quantification of the percentage of ester units in PC5 can be performed using multiple different calculation procedures. The results are extremely close regardless of the applied method (see ESI for details, Table S2). It should be noted that the difference between the values is almost negligible at 120 and $150 \circ \mathrm{C}$, while it is less than $\pm 5 \%$ at low temperatures. Accordingly, there is a perfectly linear trend of ring-opening percentage and temperature, allowing a change in ester unit percentage from ca. $50 \%$ to $80 \%$ by increasing the temperature from 50 to $100{ }^{\circ} \mathrm{C}$. Extrapolating these conditions, the polymerization of monomer $\mathbf{C 5}$ should be performed beyond $200{ }^{\circ} \mathrm{C}$ to obtain pure polyester, which is not relevant. These results are in good agreement with those previously reported in the literature concerning the trend, but also with ring-opening percentage values.

In the case of the $\mathbf{C 5 H}$ polymerization, only acetal units were observed in the polymer chains by both ${ }^{1} \mathrm{H}$ and ${ }^{13} \mathrm{C}$ NMR (see
Figure S4 and S5), as was previously reported within the literature. ${ }^{[37]}$ Moreover, unlike the other CKA monomers, molar masses were found to be very high $\left(>30,000 \mathrm{~g} \cdot \mathrm{mol}^{-1}\right)$. The sixmembered ring monomer (C6) was then polymerized at the same temperatures. The quantity of precipitated polymer was lower than previous reactions and therefore the uncertainty was higher, and thus a sample polymerized at $100^{\circ} \mathrm{C}$ was added to compensate.
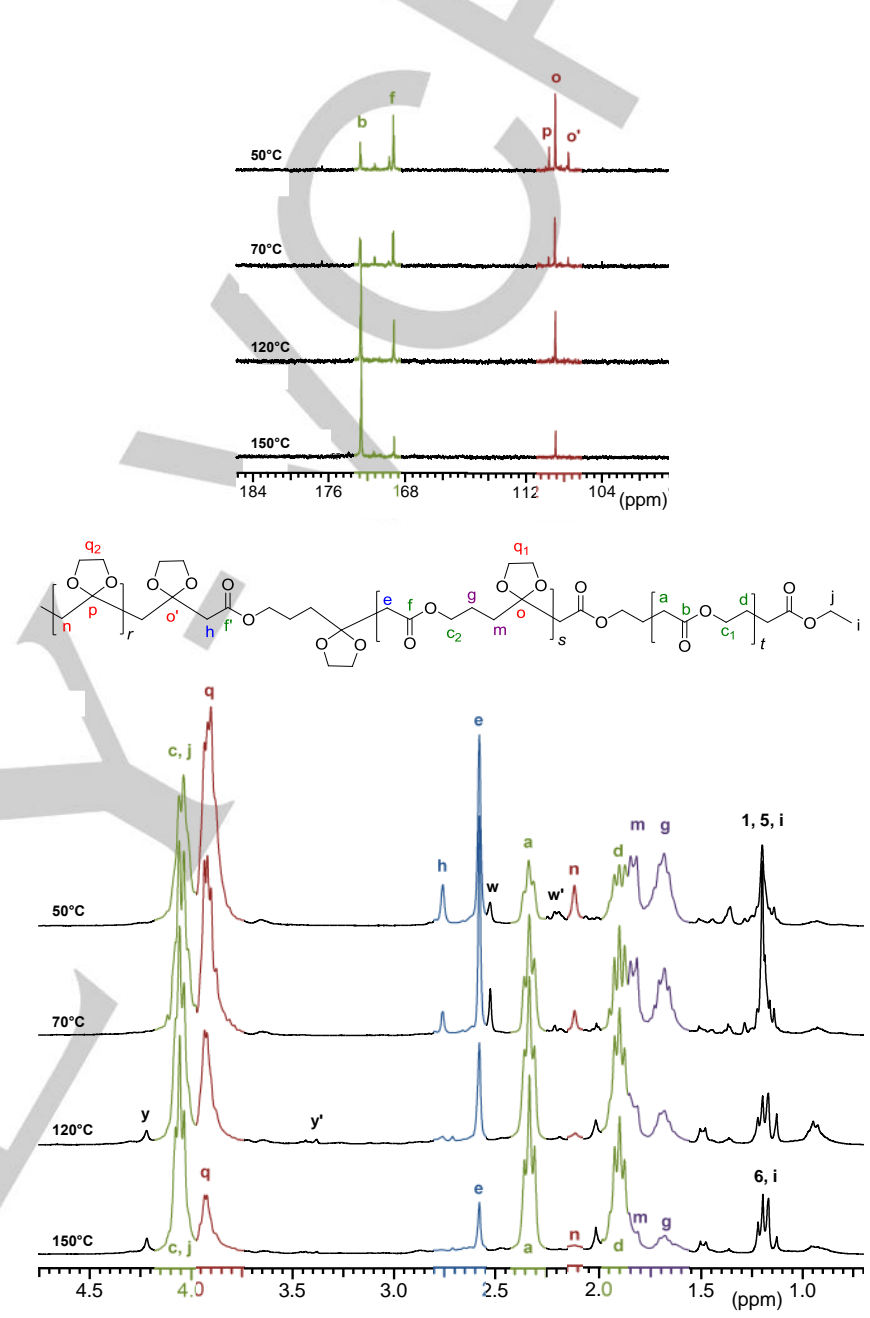

Figure 1. ${ }^{1} \mathrm{H}$ and ${ }^{13} \mathrm{C}$ NMR spectra and structure of PC5 obtained at different temperatures.

The obtained molar masses were also relatively small when compared to PC5. We noted that the ${ }^{13} \mathrm{C}$ NMR spectra had the same basic characteristics as those presented in Figure 1 (the presence of peaks corresponding to both ester and acetal units at all temperatures; an increase in the temperature leading to better ring-opening ability). However, whereas previously the ester peak intensity significantly exceeded the intensity of acetal peaks at temperatures above $120^{\circ} \mathrm{C}$, the intensities are much closer in this case. Moreover, the peak $b$ is almost absent at low temperatures (Figure S6-S8), demonstrating a lower ringopening ability of such 6-membered CKA monomer when compared to their 5-membered analogues. Nevertheless, the results still demonstrated a linear evolution in the ring-opening percentage in relation to temperature. At $130{ }^{\circ} \mathrm{C}$ we calculate 
$50-60 \%$ ester units, which is significantly below the value of $85 \%$ reported within the literature ${ }^{[11]}$ (Figure 2).

As expected from the literature data, the 7-membered ring monomer (MDO, C7) provided only polyester regardless of the temperature used (see ESI Figure S9-S10 for details).

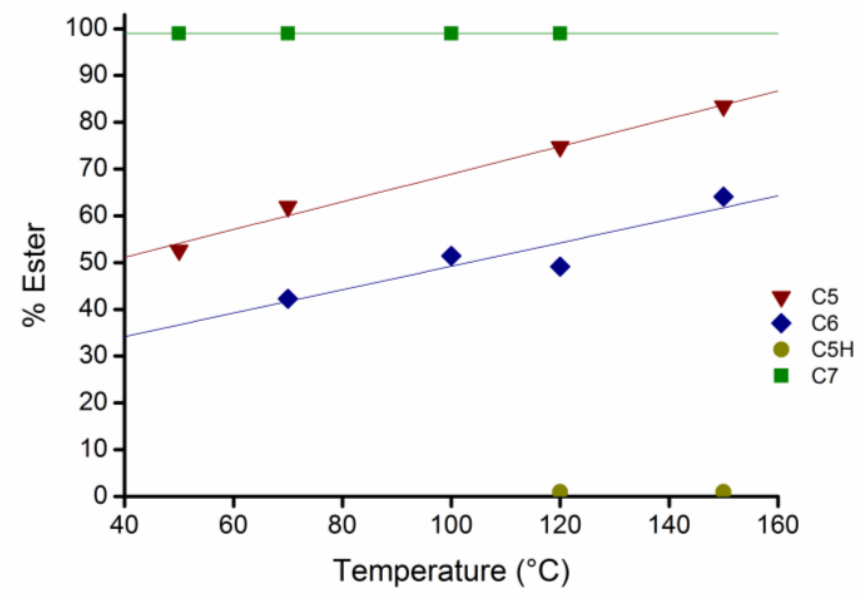

Figure 2. Ring-opening percentage as a function of temperature for the bulk polymerization of monomers C5-C7.

All of the obtained experimental data is in good agreement with the literature data. Indeed, the order for obtaining polyesters follows the trend: $\mathbf{C 5 H} \ll \mathbf{C 6}<\mathbf{C 5} \ll \mathbf{C} 7$. Nevertheless, we are now able to examine these results in detail. The 5- and 6membered monomers both have the ability to form aliphatic polyester, their ring-opening ability increasing also linearly with temperature, with an almost identical slope, whatever the monomer. It was also demonstrated that there is a clear difference in the ring-opening ability of the 5- and 6-membered ring CKA monomers, with the 6-membered CKA being less prone to ring-opening. These obtained data were subsequently used as a reference set for the following theoretical modeling.

\section{Computational study}

The different structures of the monomer and adduct radicals have been optimized by the DFT method B3LYP / 6-31G (d), commonly used for its excellent quality of results and calculation times. ${ }^{[14,38-39]}$ Moreover, this method has already been efficiently used in conformational studies of various heterocyclic rings. ${ }^{[40-41]}$ The acetalyl propagating macroradicals has been optimized by simulating the polymer chain using a methyl group (Scheme 3, $\mathbf{R 5 - R 7}$ radicals) to mimic steric hindrance without requiring significant calculation costs, while simultaneously reducing energy errors due to free rotation of the chains. In each case, the two $\mathrm{C}-\mathrm{O}$ bonds susceptible to $\beta$-scission were broken to find the transition state of lowest energy. Here we only show the values for the selected transition state. Concerning the various cyclic radicals having an aromatic substituent (R5P, R6P and R7P) the homolysis is always located on the same side as the substitution, as expected. There is only scarce experimental data for the $\beta$-scission of CKA monomers on which we can base a theoretical approach. The only values originate in a 1982 study where Ingold ${ }^{[42]}$ studied $\mathbf{1 1}$ and $\mathbf{I 2}$ (Figure 3) intermediates by
EPR and measured their $\beta$-scission rate coefficient. To determine the activation energies we used different quantum methods (i.e., UB3LYP, MO6-2X and G3(MP2)RAD) since it was shown that the particular method applied could lead to drastic differences when compared with experimental data. The results of the calculations are displayed in Figure 3. Importantly, it was observed that calculations performed using the UB3LYP method led to a lower $E_{\mathrm{a}}$ than the experiment, while calculations made with G3(MP2)RAD and MO6-2X are slightly and exceedingly higher than the experimental data, respectively. This result is not surprising as the UB3LYP method is known to generally underestimate the energy of structures. ${ }^{[43]}$ It is additionally important to note that a difference of $5-8 \mathrm{~kJ} \mathrm{~mol}^{-1}$ between the energies of activation for the two species $\mathbf{I} \mathbf{1}$ and $\mathbf{I} \mathbf{2}$ is correctly assessed by all techniques (difference between 3 and 6.5 $\left.\mathrm{kJ} \cdot \mathrm{mol}^{-1}\right)$.

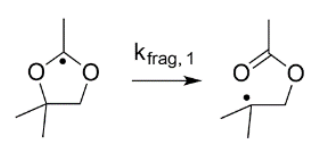

12

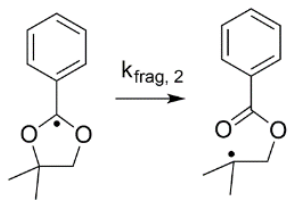

\begin{tabular}{|c|c|c|c|c|c|}
\hline \multirow{2}{*}{$\begin{array}{l}\text { Optimization } \\
\begin{array}{c}\text { Energies and } \\
\text { frequencies }\end{array}\end{array}$} & \multicolumn{3}{|c|}{ UB3LYP/6-31G(d) } & \multirow{2}{*}{$\begin{array}{l}\text { M06-2X/6- } \\
31 G(d)\end{array}$} & \multirow{2}{*}{ Exp. } \\
\hline & $\begin{array}{l}\text { UB3LYP/6- } \\
\text { 31G(d) }\end{array}$ & $\begin{array}{c}\text { UB3LYP/6- } \\
311++G(3 d f, 2 p)\end{array}$ & G3(MP2)RAD & & \\
\hline radical & \multicolumn{4}{|c|}{$E_{\mathrm{a}}$} & $E_{\mathrm{a}}$ \\
\hline 11 & 58.51 & 55.19 & 71.80 & 81.67 & 61.1 \\
\hline 12 & 63.50 & 61.67 & 74.84 & 86.60 & 66.9 \\
\hline$\left(\Delta E_{a}\right)$ & (4.99) & (6.46) & $(2.96)$ & $(5.07)$ & (5.8) \\
\hline
\end{tabular}

Figure 3. Influence of the quantum method on the activation energies of the $\beta$ scission reaction of acetalyl-based radicals and their comparison with experimental data. ${ }^{[42]}$

The values obtained with the method UB3LYP/6-31G(d) are very close but lower than the experimental values with a $3 \mathrm{~kJ} \mathrm{~mol}^{-1}$ deviation. On the other hand, the use of a larger base (i. e. 6$311++\mathrm{G}(3 \mathrm{df}, 2 \mathrm{p}))$ is not interesting as it further underestimates the energies with a deviation of 5-6 $\mathrm{kJ}^{\mathrm{mol}}{ }^{-1}$. The G3(MP2)RAD method, ${ }^{[43-44]}$ known for the quality of its absolute values, is less close to the experimental data than UB3LYP (10-11 kJ.mol ${ }^{-1}$ higher deviation). As the G3(MP2)RAD calculations require much longer and are limited to a few atoms, the UB3LYP/6$31 \mathrm{G}(\mathrm{d})$ method appeared to present the best compromise in speed/performance. We already utilized the same DFT method for other quantum calculations of CKA monomers. ${ }^{[14,45-46]}$

The activation energy values of $\beta$-scission all range between 35 and $70 \mathrm{~kJ} \mathrm{~mol}^{-1}$ (Figure 4a). All of the 7-membered CKA monomers known to polymerize exclusively by the ring-opening mechanism have an activation enthalpy lower than $40 \mathrm{~kJ} \mathrm{~mol}^{-1}$, in agreement with the previous calculations of Endo. ${ }^{[32]}$ This study also confirmed that the stabilization of the released radical is not a key parameter since there is no direct link between the percentage of ring-opening and the presence of an aromatic ring in the $\beta$ position to the cyclic acetal functionality. Moreover, these calculations also do not allow a discrimination between a partial and the absence of ring-opening. Thus far, only $\beta$ - 
scission reactions have been studied; here and within the literature. This is only a part of the complete mechanism since radical ring-opening polymerization is characterized by the competition between the $\beta$-scission and the direct addition. The reactions involved are not of the same order (unimolecular and bimolecular), and thus they are not comparable in terms of thermodynamics or activation energy, such comparisons usually being performed to identify the most-favorable reaction mechanism.

The correct method to understand the role of the different reactions is to achieve a kinetic model using not only the energy of activation but also the rate coefficients $k_{\beta}$ and $k_{\text {add,closed }}$ (Scheme 4). Indeed, to describe the macroscopic result (the ratio between the acetal and ester units in the polymer backbone) we need to compute the number of the two propagation events that occurred during the polymerization process, and thus the addition reaction after opening $k_{\text {add,open }}$ is also mandatory. Since our interest is focused on the propagation steps, we used classic initiation and termination steps and disregarded all side reactions such as chain transfer reactions that are known to occur during the polymerization of MDO, as an example. ${ }^{[4]}$ To make an accurate estimate of the addition rate coefficient of macroradicals onto monomers it is recommended to model dimers or trimers instead of unimers. ${ }^{[48]}$

However, as this work involves relatively large molecules (15-60 atoms) we thus chose to directly use the rate coefficient of simple addition reactions to limit computing time. Moreover, in the case of the addition of radicals after ring-opening, the penultimate effect was not envisioned since the previous unit is at minimum 5 atoms away. In the case of the radical addition of acetalyl-based radicals, it is assumed that the effects of steric hindrance and the specificity of such radicals are well above any potential penultimate effect. In the calculations we also did not take into account any solvent effect. Indeed, this study aimed at comparing ring-retaining and ring-opening reactions and in this case, absolute rate constants that require more complex calculations are not necessary.

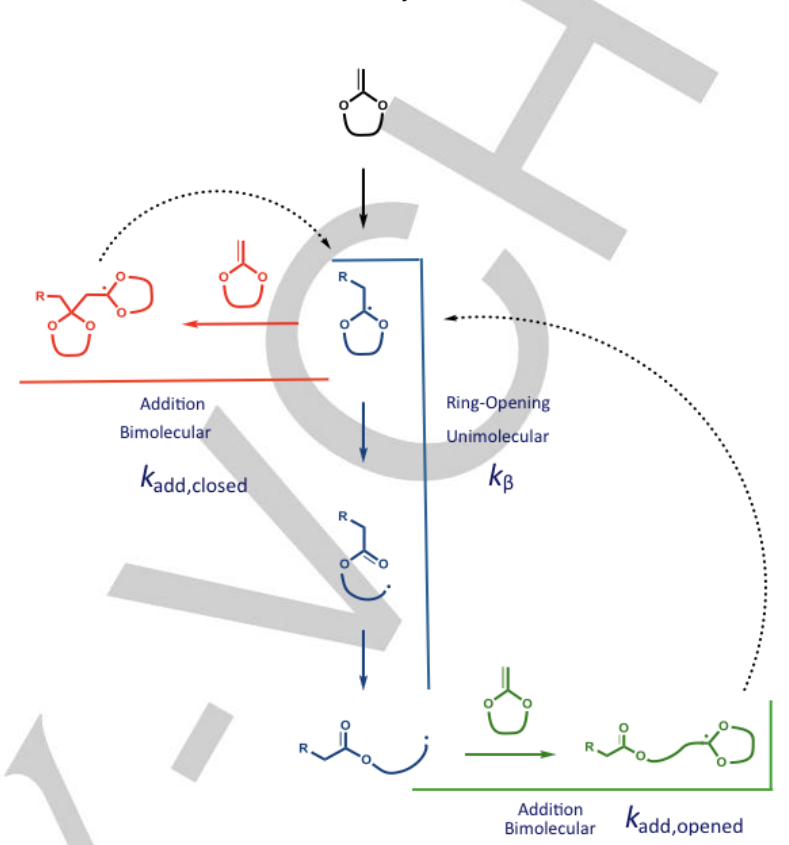

Scheme 4. Complex mechanism to theoretically determine the ester/acetal ratio in a polymer backbone. a)
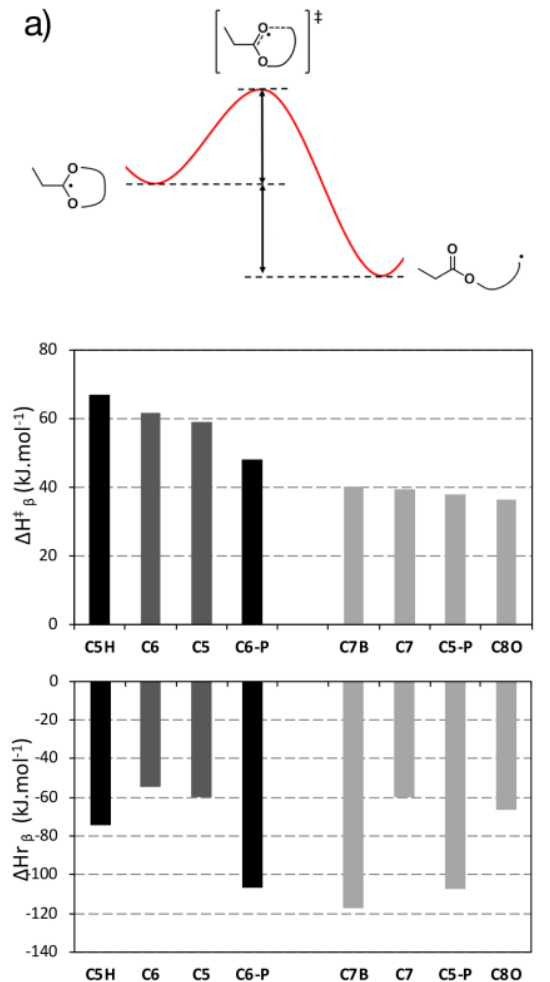

b)
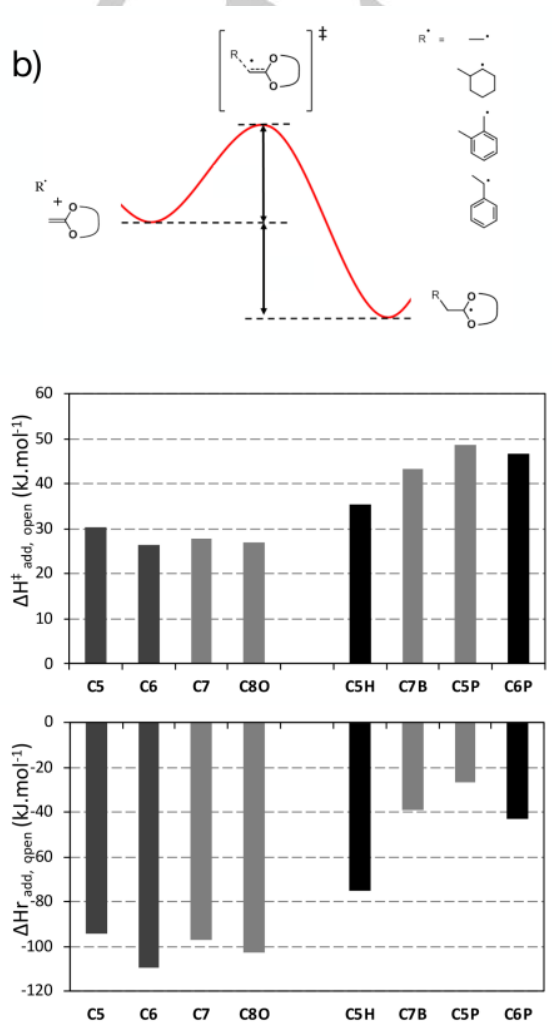

c)
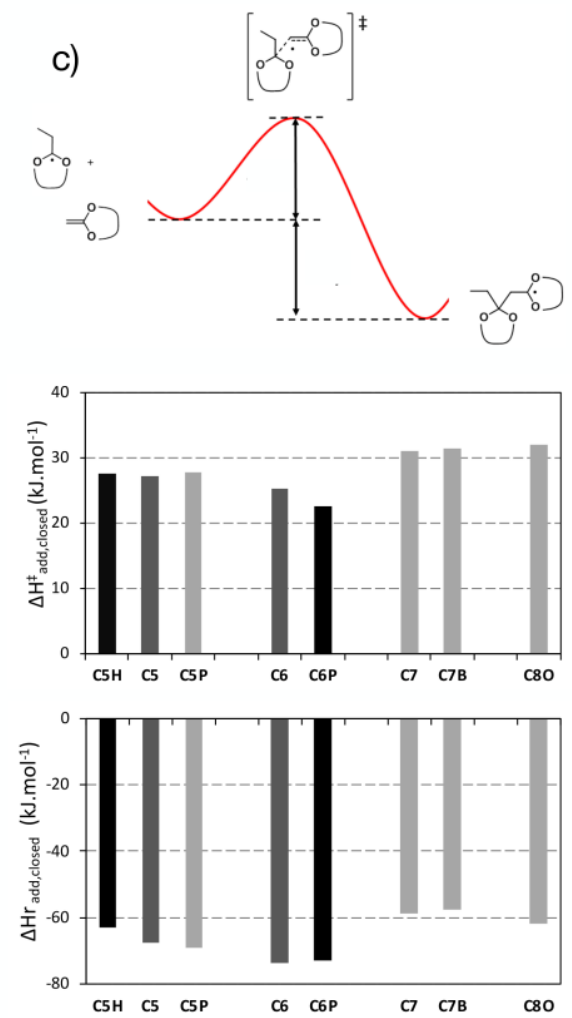
Figure 4. Enthalpies of activation $\Delta \mathrm{H}^{\ddagger}$ and reaction $\Delta \mathrm{H}_{\mathrm{r}}$ for the ring-opening reaction (left) and the different radical addition (open-type radical, middle) and acetalyl-based radical (right).

Moreover, since the polymerizations are performed in bulk, the solvent's parameters are related to the monomers themselves and are thus unknown. It is therefore beyond the scope of the manuscript to take them into account.

Concerning the addition of ring-opened radicals, the growing number of possible rotations along the length of the aliphatic chains gives access to a very large amount of possible geometries. In this way, there is too great an uncertainty in the values of calculated energies. To avoid this limitation, simple representative radicals mimicking the macroradical were modelled i.e. the ethyl radical for monomers $\mathbf{C 5}-\mathbf{C 7}$ and the styryl- or methyl-benzyl-type radical for the C5P-C7B monomers (Figure 4b). The work of Fisher and Radom ${ }^{[38]}$ which was dedicated to modeling additions of radical reactions by DFT indicates that in the case of relatively large systems the B3LYP method is appropriate and has a good compromise between time and result, also later confirmed by Moscatelli. ${ }^{[39]}$ Thus, and also to be consistent in the various calculations (addition and $\beta$ scission), this method was chosen to be applied.

The results for the addition of ring-opened radicals onto the CKA monomers are described in Figure $3 b$. Since in all cases the polarity of both adding radicals and CKA are similar, thus the rate of addition is dependent only on the stabilization of the alkyl radical (primary, secondary or tertiary alkyl radicals or the presence of a stabilizing aromatic ring). For example, the activation enthalpies of primary alkyl radicals are centered around $28 \pm 5 \mathrm{~kJ} \mathrm{~mol}^{-1}$, while are higher in the case of secondary radicals $\left(35,5 \mathrm{~kJ} \mathrm{~mol}^{-1}\right.$ for $\left.\mathbf{C} 5 \mathrm{H}\right)$. For aromatic-based monomers, the benzylic type radical has a lower activation enthalpy (43

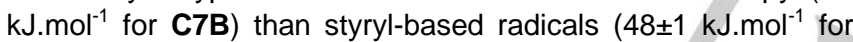
C5P - C6P). These obtained values can be compared to the values described by Fischer and Radom ${ }^{[38]}$ dealing with the addition of methyl and benzyl radicals onto vinyl ethers. In this case, activation energies of 25 and $40 \mathrm{~kJ} \mathrm{~mol}^{-1}$ were given, respectively, that are both $3 \mathrm{~kJ} \mathrm{~mol}^{-1}$ lower than the values that we obtained for CKA, which are vinyl ethers in which an additional oxygen atom is located in the a position to the vinyl functionality. Moreover, for the C5 monomer, Coote et al. ${ }^{[4]}$ proposed a value of $30 \pm 5 \mathrm{~kJ} \mathrm{~mol}^{-1}$ via high level ab initio calculations for the propagation rate coefficient that is coherent with our value. From these results we could conclude that the ring-size does not greatly affect the activation enthalpies for similar structures (C5-C6-C7 and C5P-C6P).

To our knowledge, there is no data within the literature reporting the radical addition of acetalyl-type radicals, especially onto cyclic ketene acetals. The obtained results are summarized in Figure 4c. The data shows that the calculated energy values do not differ significantly, i.e. all the activation enthalpies being centered around $27 \pm 5 \mathrm{~kJ} \mathrm{~mol}^{-1}$ and the reaction enthalpies around $-66 \pm 8 \mathrm{~kJ} \mathrm{~mol}^{-1}$. It can be observed that only the ring size seems to influence such values since $\mathbf{C 5}, \mathbf{C 5 H}$ and $\mathbf{C 5 P}$ are similar, and such behavior holds true for 6- and 7-membered ring CKA monomers. It should be noted that these values are of the same order of magnitude as the addition of primary alkyl radicals on the CKA while the acetalyl radicals here are tertiary, showing the specific reactivity of such radicals. The activation energies calculated for different reactions are not directly comparable to know whether a monomer is more likely to propagate directly or undergo $\beta$-scission. Indeed, there are different order reactions; i.e. monomolecular and bi-molecular. Therefore, kinetic modeling is required. Kinetic modeling is performed from the rate coefficients of the various reactions. These coefficients can be obtained by knowing the free energy of activation $\Delta \mathrm{G}^{\ddagger}$ or from the Eyring equation through the various components of enthalpy and entropy of activation $\left(\Delta \mathrm{H}^{\ddagger}\right.$ and $\Delta \mathrm{S}^{\ddagger}$, respectively). However, DFT methods are known to give erroneous evaluations of entropy. ${ }^{[50-51]}$

Indeed, the Gaussian software treats all vibrations like harmonic oscillators. This is correct for certain vibrations, but the entropy associated with certain hindered rotations is not properly taken into account. In the cyclic ketal intermediate, such hindered rotations are important and thus $\Delta S^{\ddagger}$ leads to erroneous results. The use of the Arrhenius equation simplifies the problem. Indeed, the pre-exponential factor A contains the component of entropy and its value is rather similar for the same type of reaction in a narrow range of temperatures. ${ }^{[38]}$ For the addition of open radicals, the values of $\log A$ that are recommended are $7.5,8$, and 8.5 depending on whether the groups are tertiary, secondary or primary, respectively. ${ }^{[38]}$ However, no value for the addition of tertiary acetalyl-based radicals exist in the literature, the presence of more oxygen atoms appears to increase the value of $\log A$ in different cases. ${ }^{[38]}$ Therefore, we chose to use the average value of 8 for such acetalyl radicals. In the case of the ring-opening reaction, it is recommended to take a $\log A$ value of $13 .{ }^{[42,52]}$ Kinetic modeling was then performed using PREDICI software. This software is based on the deterministic Galerkin h-p-method has been widely used for describing molecular weight distributions and polymer reaction kinetics for a large range of polymer processes. ${ }^{[4-46,53]}$ The kinetic scheme is depicted in Scheme 5 while the coefficients, hypotheses and applied approximations are detailed within the ESI (Table S7 and S8).

The propagating species are simply either closed or open type. We assumed that the rate coefficients are independent to the chain length. This assumption is more relevant for the propagation step than for the termination step, since diffusional limitations are more important in the latter case. ${ }^{[54]}$ 

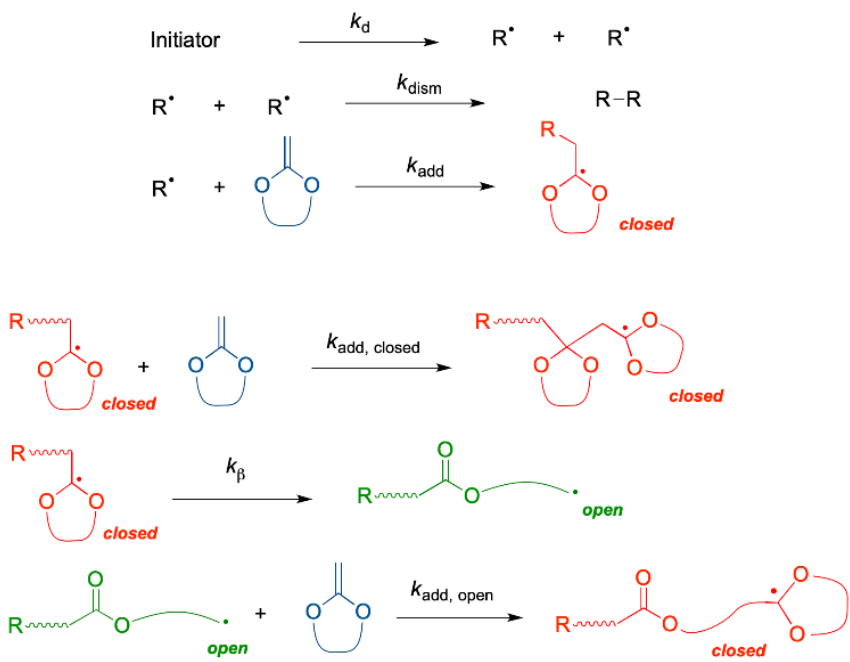

$$
\begin{array}{ll}
\text { closed }+ \text { open } & \stackrel{k_{\mathrm{t}, \text { mixed }}}{\longrightarrow} \\
\text { closed }+ \text { closed } \stackrel{k_{\mathrm{t}, \text { closed }}}{\longrightarrow} \text { Dead Polymer chains } \\
\text { open }+ \text { open } \stackrel{k_{\mathrm{t} \text {, open }}}{\longrightarrow}
\end{array}
$$

Scheme 5. Kinetic model used in the PREDICI software.

It has been already stated that the chain length was not taken into account because the larger molecular size requires too much computation time. Moreover, by modeling propagating radicals in the form of dimers or trimers, the penultimate unit would be different according to the previous addition (e.g. closed-closed, open-closed, closed-open or open-open dimers), which would complexify the system and thus would require many additional calculations that are not necessarily useful in this particular case. Counters have been inserted into the model to quantify the amount of ester and acetal functions introduced in the copolymer. The acetal counter was increased when there is an addition of a closed-type radical. The ester counter increased when ring-opening occurred. In that way, it does not include counting when there is an addition of an open-type radical since the resulting chain end is then of a closed type and can therefore react in both ways. At the end of the modelling, the number of acetal and ester counters were recorded and compared to determine the percentage of ester units in the final polymer backbone.

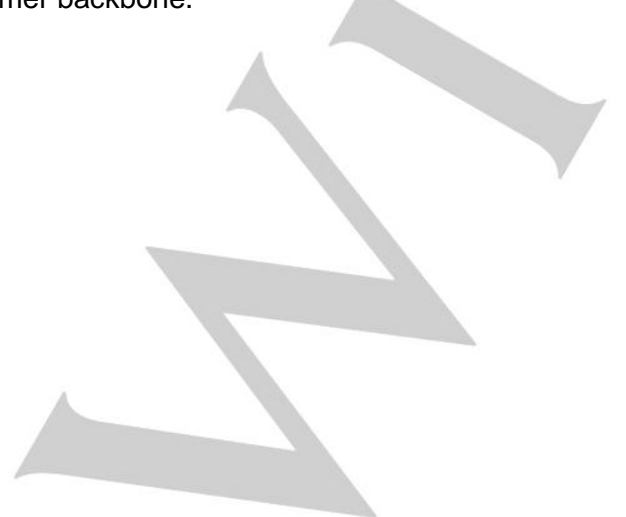

a)
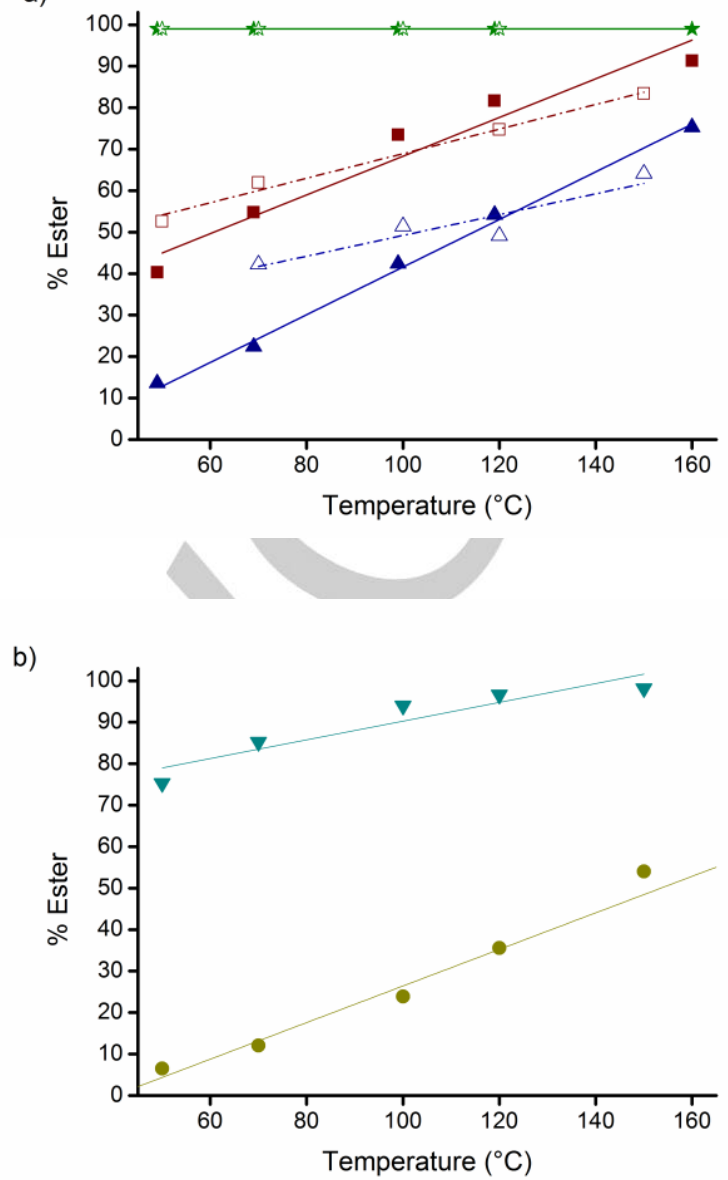

Figure 5. a) Theoretical (plain symbol and straight line) and experimenta (empty symbol and dashed-dotted line) ring opening percentage as a function

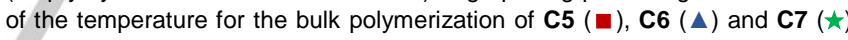
monomers; b) Theoretical ring opening percentage as a function of the temperature for the bulk polymerization of $\mathbf{C 5 H}(\bullet)$ and $\mathbf{C 6 P}(\boldsymbol{\nabla})$ monomers.

The result of the modeling is depicted in Figure 5. A good agreement between the experimental values and those obtained from the model can be observed in the case of simple monomers C5, C6 and C7. Indeed, Figure 5 shows relatively identical behavior between the simulation and the experimental observations for these monomers; $\mathbf{C 7}$ monomer gives only ester units at $50^{\circ} \mathrm{C}$ while $\mathbf{C 5}$ and $\mathbf{C 6}$ form copolymers. Moreover, the difference in ability between $\mathbf{C 5}$ and $\mathbf{C 6}$ to form polyesters is also in good agreement with the experimental results, with $75 \%$ and $45 \%$ polyester at $100{ }^{\circ} \mathrm{C}$, respectively, (on average $20 \%$ more for $\mathbf{C 5}$ than $\mathbf{C 6}$ ) and an increase in percentage of polyester with an increase in temperature. In contrast, the slope defining the increase in ester percentage depending on the temperature is slightly steeper for the simulation results in comparison to the experimental data. This discrepancy could originate from the assumptions made regarding chain length independency of the various kinetic rate coefficients. Nevertheless, this modeling approach provides an interesting and relatively accurate tool to describe the reactivity of CKA monomers. We additionally performed such modeling on more complex monomers. With C5P, C7B and C80 the model gives complete ring-opening, in accordance to the literature data. ${ }^{[10]}$ In contrast to experimental 
data, the model predicts that $\mathbf{C} 5 \mathbf{H}$ should contain some ester units even if such a monomer has a lower tendency to ringopen. ${ }^{[37]}$ The results obtained for $\mathbf{C 6 P}$ (Figure 6) is not in agreement with the literature data ${ }^{[34]}$ since the modeling showed an enhancement in the ring-opening ability in comparison to the C6 CKA monomer ( $75 \%$ instead of $25 \%$ at $70{ }^{\circ} \mathrm{C}$ ).

The discrepancy between the theoretical results and the literature data can be explained by cationic polymerization that was not inhibited. ${ }^{[34]}$ This explanation is further supported by a study on C6 CKA derivatives bearing methyl groups on the carbon in the a position relative to the cyclic acetal functionality. In that case, using base to prevent cationic polymerization, they obtained $89 \%$ ester units at $120^{\circ} \mathrm{C} .{ }^{[55]}$ Another parameter that could be used to increase the ring-opening ability of CKA monomers is to decrease the monomer concentration in order to favor the unimolecular reaction compared to the undesired bimolecular acetal propagation. To simulate the monomer dilution, the ring-opening percentage of the $\mathbf{C 6}$ monomer at different initial monomer concentrations and temperatures was modeled (Figure 6). As expected, an evolution towards the production of more polyester at high dilution was observed.

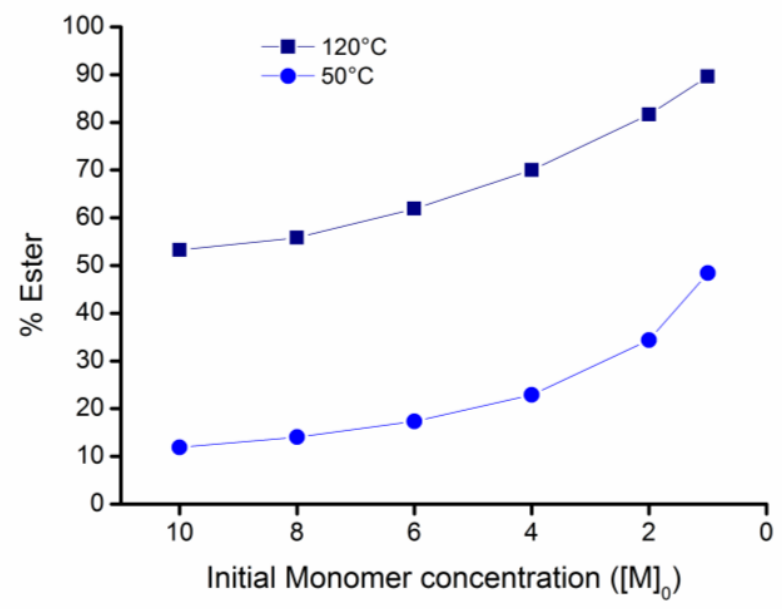

Figure 6. Theoretical ring-opening percentage as a function of temperature and initial monomer concentration $[\mathrm{M}]_{0}$ for the $\mathbf{C 6}$ monomer.

On the other hand, the curves level out at the limit values of around 10 and $50 \%$ polyester at 50 and $120^{\circ} \mathrm{C}$, respectively for an initial monomer concentration of $8 \mathrm{~mol}^{-\mathrm{L}^{-1}}$, confirming that the value we used in previous simulations is representative of the bulk polymerization. Although relatively simple, the kinetic model we have developed appears robust enough to allow an approximation of the behavior of a CKA monomer. The model requires the determination of three activation energies to investigate the reactivity of new CKA monomers. According to the calculations performed for the addition reactions, it appeared possible to approximate the rate of acetal-based addition according to the monomer ring size and the rate of addition of open-type alkyl radicals by using previously determined values for the addition of model alkyl radicals onto simple CKA monomers.

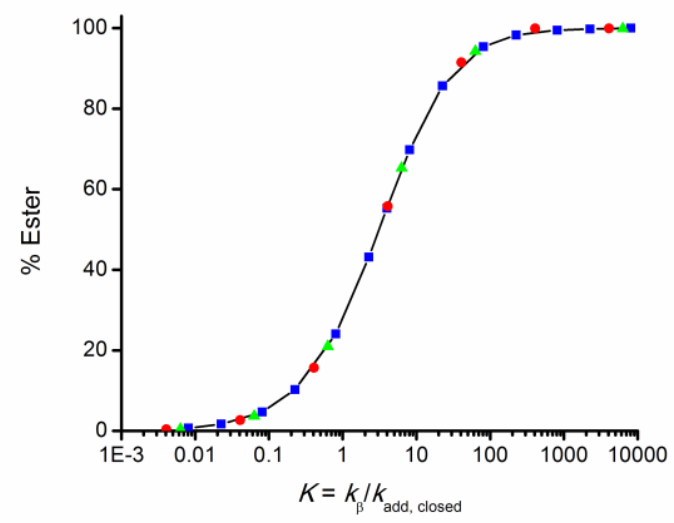

Figure 7. Theoretical ring-opening percentage as a function of the paramete $K=k_{\beta} / k_{\text {add,closed }}$ see Scheme 4 for the definition of the rate coefficients; ( $\left.\mathbf{\square}\right) \mathbf{C 6}$ monomer at $70{ }^{\circ} \mathrm{C}$; (O) $\mathbf{C 6}$ monomer at $120^{\circ} \mathrm{C} ;(\Delta) \mathbf{C 8 0}$ monomer at $70{ }^{\circ} \mathrm{C}$.

Using such an approach, only the $\beta$-scission of the intermediate acetalyl-radical has to be calculated. Secondly, we performed the modeling using the parameters used for $\mathbf{C 6}$ and $\mathbf{C 8 O}$ monomers (i.e. the monomers that have the highest and lowest activation energies for ring-opening) and determined the percentage of ester units in the polymer backbone versus a constant $K$ that is determined by the ratio $k_{\beta} / k_{\text {add }}$, closed (Figure 7). Interestingly all the data followed a master curve. A threshold value of $1 \times 10^{3}$ seems mandatory to obtain pure polyester.

Table 1. Upper values for the enthalpy of activation for the ring-opening reaction to obtain pure polyester according to CKA ring size

\begin{tabular}{ccc}
\hline $\begin{array}{c}\text { CKA Monomer } \\
\text { Size }\end{array}$ & $\begin{array}{c}\text { Average } \\
\Delta \mathrm{H}^{\ddagger}{ }_{\text {add, closed }} \\
\left(\mathrm{kJ} . \mathrm{mol}^{-1}\right)\end{array}$ & $\begin{array}{c}\Delta \mathrm{H}^{\ddagger}{ }_{\beta} \max \\
\left(\mathrm{kJ} \cdot \mathrm{mol}^{-1}\right)\end{array}$ \\
\hline 5 & 27 & 40 \\
6 & 24 & 37 \\
7 & 31 & 44 \\
\hline
\end{tabular}

Using this threshold and the values of the pre-exponential factors, we can estimate that a CKA monomer should have a difference between the activation energies of ring-opening and addition of the ring-retaining acetalyl lower than $13-15 \mathrm{~kJ}^{\mathrm{mol}}{ }^{-1}$ to efficiently obtain polyester. If we assume that the radical addition of the acetalyl intermediate is only determined by the ring size, this approach gives an upper limit for the $\beta$-scission reaction depending of the ring size (Table 1).

\section{Conclusion}

The competitive process of ring-retaining propagation is a major obstacle in the development of efficient CKA monomers. In this study we investigated this key parameter by a combination of experimental and theoretical approaches. First, it was demonstrated that the ratio between ester and acetal units in the polymer backbone is linearly correlated to the polymerization 
temperature, with the ease of ring-opening following the order C7 > C5 > C6. DFT calculations of the ring-opening reaction showed that complete ring-opening behavior could be obtained with a $\Delta \mathrm{H}^{\ddagger}<40 \mathrm{~kJ} \cdot \mathrm{mol}^{-1}$ but it was not possible to discriminate or quantify the behavior for other monomers. By further utilizing the kinetic modeling software PREDICl the behavior of CKA monomers was able to be rationalized by considering the impact of the addition of acetalyl and open-type radicals onto the CKA monomer.

Although relatively simple, the developed kinetic model is based on relevant values and seems robust enough to allow for an approximation of the behavior of a CKA monomer after completing the necessary energy calculations. Furthermore, this model allows an attempt at the rationalization of behavioral differences between the monomers through structure-reactivity relationships. The analysis of molecular orbitals is also predicted to be an excellent method for this because it can separate the contributions due to hyper-conjugation and those due to other energy contributions such as steric and electrostatic interactions. This will be the subject of a subsequent study.

\section{Acknowledgements}

We thank the French Ministry of Research for the financial support of the PhD thesis of A.T and the CNRS and the French National Research Agency (ANR-15-CE08-0019 and ANR-11JS08-0005). This work was also supported by the 'Centre Régional de Compétences en Modélisation Moléculaire de Marseille'. Arkema is acknowledged for providing DEAB.

Keywords: DFT Calculations • Polyester • Radical polymerization $\bullet$ Cyclic Ketene Acetals $•$ Kinetic modelling

[1] M. Cole, P. Lindeque, C. Halsband, T. S. Galloway, Marine Pollution Bulletin 2011, 62, 2588-2597.

[2] M. Eriksen, L. C. M. Lebreton, H. S. Carson, M. Thiel, C. J. Moore, J. C. Borerro, F. Galgani, P. G. Ryan, J. Reisser, Plos One 2014, 9.

[3] R. Geyer, J. R. Jambeck, K. L. Law, Science Advances 2017, 3.

[4] E. Castro-Aguirre, F. Iniguez-Franco, H. Samsudin, X. Fang, R. Auras, Advanced Drug Delivery Reviews 2016, 107, 333-366.

[5] K. M. Nampoothiri, N. R. Nair, R. P. John, Bioresource Technology 2010, 101, 8493-8501.

[6] C. Vilela, A. F. Sousa, A. C. Fonseca, A. C. Serra, J. F. J. Coelho, C. S. R. Freire, A. J. D. Silvestre, Polymer Chemistry 2014, 5, 3119-3141.

[7] A. C. Albertsson, I. K. Varma, Degradable Aliphatic Polyesters 2002, 157, 1-40.

[8] V. Delplace, J. Nicolas, Nature Chemistry 2015, 7, 771784.

[9] S. Agarwal, Polymer Chemistry 2010, 1, 953-964

[10] A. Tardy, J. Nicolas, D. Gigmes, C. Lefay, Y. Guillaneuf, Chem. Rev. 2017, 117, 1319-1406.

[11] W. J. Bailey, Z. Ni, S. R. Wu, Journal of Polymer Science Part a-Polymer Chemistry 1982, 20, 3021-3030.

[12] C. A. Bell, G. G. Hedir, R. K. O'Reilly, A. P. Dove, Polymer Chemistry 2015, 6, 7447-7454.

[13] S. Maji, M. Zheng, S. Agarwal, Macromolecular Chemistry and Physics 2011, 212, 2573-2582.

[14] A. Tardy, J. C. Honore, J. Tran, D. Siri, V. Delplace, I. Bataille, D. Letourneur, J. Perrier, C. Nicoletti, M. Maresca, C. Lefay, D. Gigmes, J. Nicolas, Y. Guillaneuf, Angewandte Chemie-International Edition 2017, 56, 16515-16520.
[15] J. Undin, A. Finne-Wistrand, A.-C. Albertsson, Biomacromolecules 2013, 14, 2095-2102.

[16] G. X. Dai, Q. Y. Xie, S. S. Chen, C. F. Ma, G. Z. Zhang, Progress in Organic Coatings 2018, 124, 55-60.

[17] V. Delplace, A. Tardy, S. Harrisson, S. Mura, D. Gigmes Y. Guillaneuf, J. Nicolas, Biomacromolecules 2013, 14, 3769-3779.

[18] E. Guegain, J. P. Michel, T. Boissenot, J. Nicolase, Macromolecules 2018, 51, 724-736.

[19] Y. F. Shi, H. Schmalz, S. Agarwal, Polymer Chemistry 2015, 6, 6409-6415.

[20] U. Wais, L. R. Chennamaneni, P. Thoniyot, H. F. Zhang, A W. Jackson, Polymer Chemistry 2018, 9.

[21] T. Zeng, W. You, G. Chen, X. Nie, Z. Zhang, L. Xia, C. Hong, C. Chen, Y. You, iScience 2020, 23, 100904.

[22] J. H. Ko, T. Terashima, M. Sawamoto, H. D. Maynard, Macromolecules 2017, 50, 9222-9232.

[23] G. X. Dai, Q. Y. Xie, C. F. Ma, G. Z. Zhang, Acs Applied Materials \& Interfaces 2019, 11, 11947-11953.

[24] Q. Y. Xie, Q. N. Xie, J. S. Pan, C. F. Ma, G. Z. Zhang, Acs Applied Materials \& Interfaces 2018, 10, 11213-11220.

[25] E. Guegain, J. Tran, Q. Deguettes, J. Nicolas, Chemical Science 2018, 9, 8291-8306.

[26] E. Guegain, C. Zhu, E. Giovanardi, J. Nicolas, Macromolecules 2019, 52, 3612-3624. A. W. Jackson, L. R. Chennamaneni, P. Thoniyot, European Polymer Journal 2020, 122. S. Komatsu, T. A. Asoh, R. Ishihara, A. Kikuchi, Polymer 2017, 130, 68-73.

G. G. Hedir, M. C. Arno, M. Langlais, J. T. Husband, R. K. O'Reilly, A. P. Dove, Angewandte Chemie-International Edition 2017, 56, 9178-9182.

[30] G. Hedir, C. Stubbs, P. Aston, A. P. Dove, M. I. Gibson, Acs Macro Letters 2017, 6, 1404-1408.

[31] D. Gigmes, P. H. M. Van Steenberge, D. Siri, D. R. D'Hooge, Y. Guillaneuf, C. Lefay, Macromolecular Rapid Communications 2018, 39 .

[32] B. Ochiai, T. Endo, J. Pol. Sci.: Part A: Polym. Chem. 2007, 45, 2827-2834.

[33] J. B. Lena, A. M. Van Herk, Industrial \& Engineering Chemistry Research 2019, 58, 20923-20931.

[34] T. Schulze, E. Klemm, Angew. Makromol. Chem. 1995, 229, 123.

[35] W. J. Bailey, S.-R. Wu, Z. Ni, Makromol. Chem. 1982, 183, 1913-1920.

[36] I. Cho, M. S. Gong, Journal of Polymer Science Part CPolymer Letters 1982, 20, 361-364.

[37] T. Schultze, J. Letsch, E. Klemm, Journal of Polymer Science: Part A Polymer Chemistry 1996, 34, 81-87.

[38] H. Fischer, L. Radom, Angewandte Chemie International Edition 2001, 40, 1340-1371.

[39] D. Moscatelli, M. Dossi, C. Cavallotti, G. Storti, The Journal of Physical Chemistry A 2011, 115, 52-62.

[40] E. Taskinen, Structural Chemistry 2000, 11, 283-291.

[41] E. Taskinen, Structural Chemistry 2001, 12, 419-429.

[42] L. R. C. Barclay, D. Griller, K. U. Ingold, Journal of the American Chemical Society 1982, 104, 4399-4403.

[43] E. I. Izgorodina, D. R. B. Brittain, J. L. Hodgson, E. H. Krenske, C. Y. Lin, M. Namazian, M. L. Coote, Journal of Physical Chemistry A 2007, 111, 10754-10768.

[44] E. I. Izgorodina, M. L. Coote, L. Radom, Journal of Physical Chemistry A 2005, 109, 7558-7566.

[45] A. Tardy, J.-C. Honoré, D. Siri, J. Nicolas, D. Gigmes, C Lefay, Y. Guillaneuf, Polymer Chemistry 2017, 8, 51395147.

[46] A. Tardy, V. Delplace, D. Siri, C. Lefay, S. Harrisson, B. D. A. Pereira, L. Charles, D. Gigmes, J. Nicolas, Y. Guillaneuf, Polymer Chemistry 2013, 4, 4776-4787.

[47] S. Jin, K. E. Gonsalves, Macromolecules 1997, 30, $3104-$ 3106.

[48] E. I. Izgorodina, M. L. Coote, Chemical Physics 2006, 324, 96-110.

[49] G. E. Roberts, M. L. Coote, J. P. A. Heuts, L. M. Morris, T. P. Davis, Macromolecules 1999, 32, 1332-1340.

[50] C. J. Cramer, J. Wiley \& Sons, 2004, pp. 375-377. 
[51] C. Y. Lin, E. I. Izgorodina, M. L. Coote, Journal of Physical Chemistry A 2008, 112, 1956-1964.

[52] S. W. Benson, Thermochemical Kinetics, Wiley, New York, 1976.

[53] M. Wulkow, Macromolecular Reaction Engineering 2008, 2, 461-494.

[54] D. R. D'hooge, P. H. M. Van Steenberge, M.-F. Reyniers,

G. B. Marin, Progress in Polymer Science 2016, 58, 59-89. N. Zhende, Bailey, J. W, Acta Polymerica Sinica 1987, 1 , 379-383. 


\section{Entry for the Table of Contents}

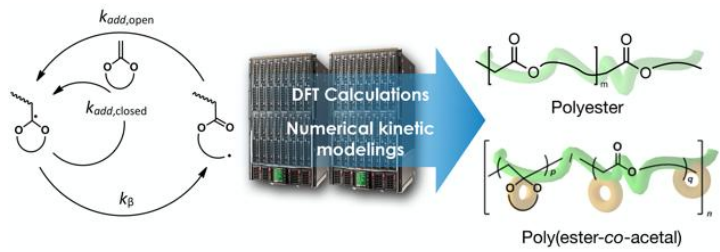

Draw me a CKA: the radical ring-opening polymerization of model CKA monomers was investigated using quantum calculations and kinetic modeling. The results were supported by a detailed experimental study. The new methodology we propose allows to predict in silico the efficiency of CKA monomers to prepare polyester via a radical pathway. 